\title{
Opportunistic Forwarding Throughout Customers or Sellers in Shopping Mall Environments
}

\author{
Adriano Galati and Karim Djemame \\ Collaborative Systems and Performance Group \\ School of Computing \\ University of Leeds \\ LS2 9JT, UK \\ Email: \{a.galati, k.djemame\}@leeds.ac.uk
}

\author{
Chris Greenhalgh \\ Mixed Reality Lab \\ School of Computer Science \\ University of Nottingham \\ NG8 1BB, UK \\ Email: cmg@cs.nott.ac.uk
}

\begin{abstract}
In this paper, we seek to improve understanding of human mobility patterns in environments having definite and highly organized structure, such as shopping malls. We present a method to identify individuals expressing different mobility patterns. Besides, to understand better the role of groups of message carriers expressing different mobility patterns, we performed simulations of a derivative of the Epidemic protocol with real-world mobility traces, which distinguishes between two groups of carriers, and entrusts messages through either one or the other. We discuss the implications of our results and make recommendations to guide the design of ad-hoc forwarding algorithms for delay tolerant mobile ad-hoc networks in shopping mall environments and to help modeling realistic simulation scenarios.
\end{abstract}

\section{INTRODUCTION}

Several studies have been performed to analyse nodes' movement in different settings: a conference environment involving conference attendees at Infocom [3], in research labs and universities in Cambridge [4], and MIT [5] involving researchers and students, in the campuses WiFi access network of UCSD (University of California, San Diego) [6], Dartmouth College [7], and ETH Zurich [8], during a Paris roller blading event [9], in an entertainment theme park [11], and in a typical office environment [12]. Although there is a range of useful data sets available no one deals with shopping malls in particular. Therefore, to design and test our network application for such an environment we have conducted a field trial aiming to gather data about contacts between devices carried by humans in a shopping mall. This data set includes handheld Bluetooth devices employed in a smaller scale environment with short granularity for six days. In this paper, which follows from our previous works [1], [2], we study relationships between two people in shopping mall environments. We provide a methodology to identify devices carried by visitors/customers and devices carried by shopping mall related people based on pure contact duration, inter-contact time and frequency. Such a method can also be employed in different structured environments. We define as structured environment, a scenario having definite and highly organised structure, where people are organised by characteristic patterns of relationship and mobility. Finally, we have conducted an initial study to understand better the potential roles of customers and sellers in forwarding messages in shopping mall environments. For that, we evaluate the performance of two semi-Epidemic protocols with our real-world mobility traces, which forward messages exclusively to customers or sellers. The paper is structured as follows: in Section II, we present a method to identify devices carried by customers and sellers in shopping malls; in Section III we present our initial simulation results of two semi-Epidemic forwarding protocols with real-world mobility traces and Section IV concludes the paper.

\section{Identifying CUstomers'and Sellers' Devices}

From the collected contact traces, we cannot straightaway assume external contacts as between sellers and customers because they can occur between sellers and any other device. Namely, external devices do not necessarily identify customers; they could be sellers' personal devices. Here, we provide a methodology to identify devices carried by visitors/customers and devices carried by shopping mall related people based on pure contact duration, inter-contact time and frequency. Namely, if an internal device spends long time in contact with an external one or they see each other very often, then, we assume that they are in "working relationship". As such they have specific duties and relatively predictable mobility. Figure 1 plots the correlation between the number of contacts and the contact durations of each device with our smartphones, day by day, distinguishing between internal and external devices. Contact duration is a single set of consecutive sightings of the same node, i.e. a presumed period of continuous contact. In this plot we consider "internal-like" nodes which are external devices behaving like internals. They express contact durations longer than three hours or number of contacts bigger than 20 (contact durations are derived from contiguous data $\operatorname{logs}$ ). We conjecture that they show contacts with devices carried by other sellers or shopping mall employees which were not part of the experiment. To identify such devices we plot the correlation between the number of contacts and the longest contact duration of each device with all our smartphones in Figure 2. Here, we can see two clusters of devices: externals on the left bottom and internals on the right bottom of the quadrant. We infer that people in the first cluster do not spend more than roughly two hours in the 


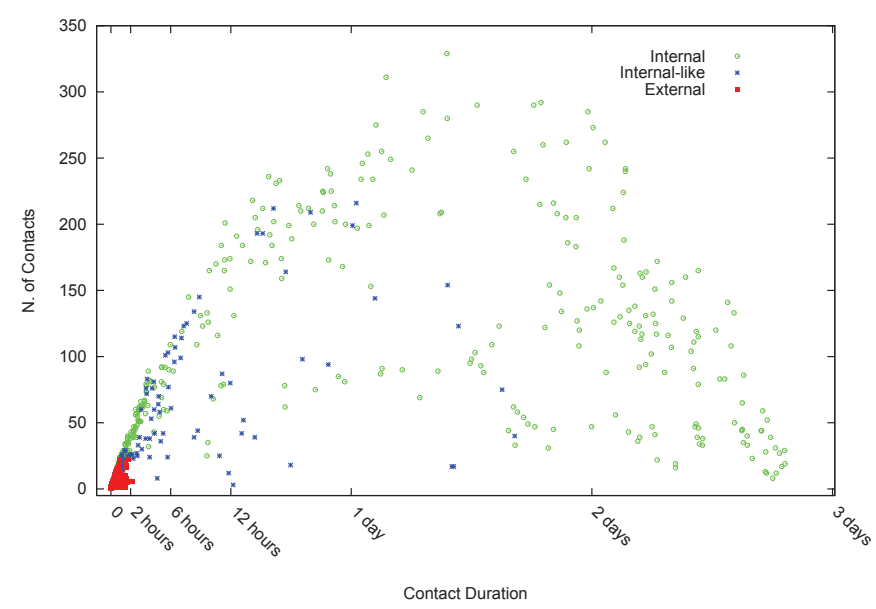

Fig. 1: The number of contacts and contact durations of each device with all the internal devices

shopping mall. Thus, we can infer that all the nodes falling in this cluster are likely to be customers. Only 9 external devices out of 752 perform higher contact duration and number of contacts. The majority of the remaining individuals fall in the second population which means that they tend to spend long time "together" and meet each other more often. That is expected sellers' and shopping mall employees' behaviour since neighbouring sellers tend to be in contact for long time. These results show a different distribution plot with respect to the previous studies [10], [13]. Furthermore, we analyze the correlation between the longest inter-contact time and the number of contacts with each external device, day by day, to filter external devices. Inter-contact time is the elapsed time between two non-consecutive sightings of the same node. We are only considering external devices in the left cluster of Figure 2. In this next step 22 external devices out of 743 have a maximum inter-contact time longer than 3 hours (within a single day). The vast majority of these devices have inter-contact time lower than one hour. After three hours the frequency decreases drastically. We then classify external devices with inter-contact time smaller than three hours as likely to be carried by customers.

\section{FORWARDING THROUGHOUT CUSTOMERS OR SELLERS}

In this section we evaluate the performance of the two semiEpidemic protocols with the mobility traces of our data set. These are identical to the Epidemic protocol except that they forward messages exclusively to customers or sellers at the intermediate hops. In our simulations we assume two nodes are also in contact with each other if they are both detected in a single round of Bluetooth discovery. Unfortunately, this assumption introduces inaccuracies. On one hand, it is overly optimistic, since two devices in sight of the same internal node may still be out of range of each other. On the other hand, the data might omit connection opportunities, since two devices may be within each other's range and not in sight of an internal node, and this contact would not be logged. We

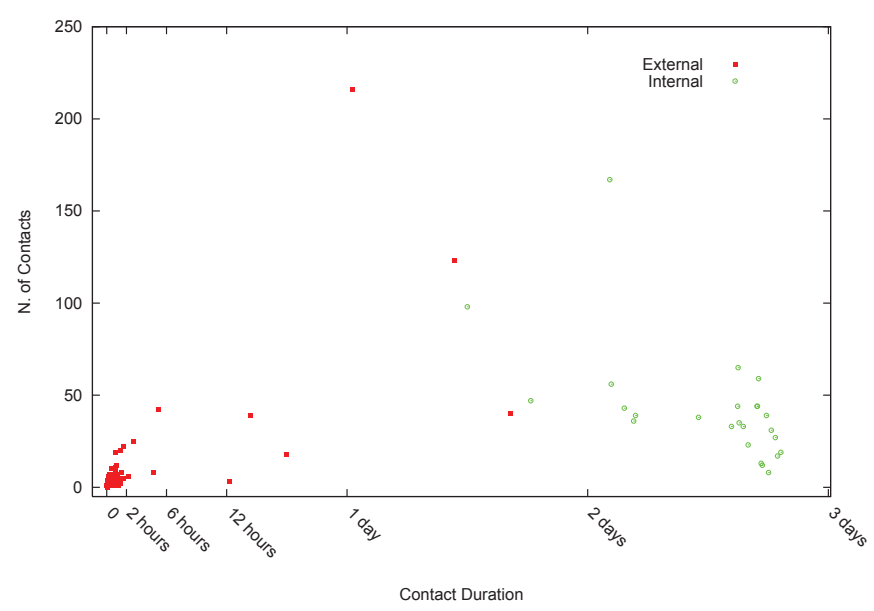

Fig. 2: Number of contacts versus the longest contact durations for internal and external devices

evaluated the performance of the routing protocols by sending 1000 messages. The minimum interval between the generation of two subsequent messages is equal to 0.1 seconds, as long as the chosen recipient node is different from the sender. The generation of messages with such a frequency produces a certain network load which will stress the network itself and allows network protocols to show better the capability of network protocols in routing data. The sender and recipient of each message were randomly chosen among all of the nodes following a uniform distribution. We run equal number of simulations with each combination of customers and sellers as senders and receivers; this was sufficient to determine a $95 \%$ confidence interval using a t-distribution.

The performance of the Epidemic protocol is strictly correlated to the connectedness of end-to-end paths with hosts with infinite buffer capacity. By comparing these results we aim to expose differences in forwarding data through customers versus sellers in such environments. In the Figure 3 and Figure 4 we plot respectively the delivery ratio and the average delay of the four separate combinations of customers and sellers as sender and receiver for the two routing protocols. Combinations are identified in the figures using the syntax $[C \mid S]([C \mid S]$ to $[C \mid S])$ where the first field identifies whether the semi-Epidemic protocol forwards messages through customers $\mathrm{C}$ or sellers S. The Figures 3 and 4 show the performance of the semi-Epidemic routing protocols with two different buffer capacities. The white and the red boxes show the results when the buffer capacity of the nodes is $10 \%$ and $20 \%$, respectively, of the number of the messages generated. As expected higher buffer capacities lead to high delivery ratios, and the impact of mobility is similar in both buffer capacities. The delivery ratio in the left part of Figure 3, that is CtoC and CtoS, is slightly higher if messages are delivered through sellers. In contrast, in the right part of Figure 3 messages routed through sellers result in lower delivery ratios. We conjecture that this is mainly because of the limited buffer capacity of each node and the different mobility pattern and relatively small number of sellers with respect to 


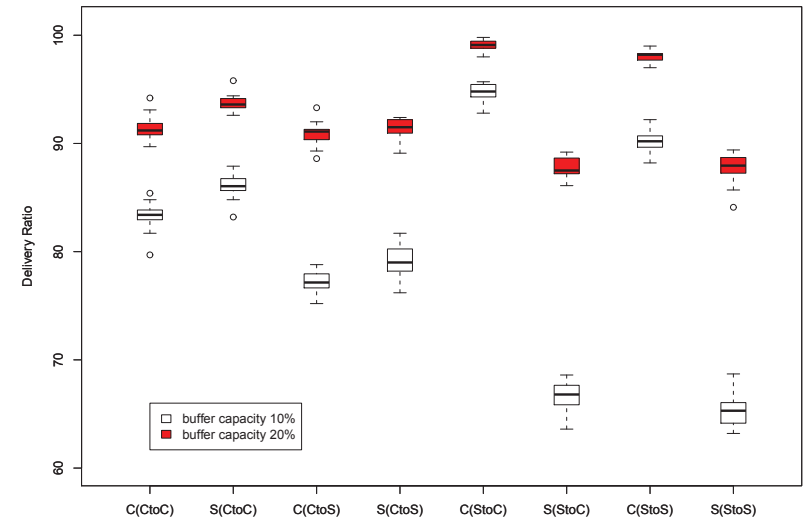

Fig. 3: Boxplot of the delivery ratio for the semi-Epidemic routing protocols.

customers. Thus, the network becomes more overloaded when messages are generated by sellers and routed throughout them. This leads to buffer overflow and message loss. This is backed up by cases $S(S t o C)$ and $S(S t o S)$ where increasing buffer capacity has the biggest impact on the performance of the routing protocols. We believe that $\mathrm{C}(\mathrm{CtOC})$ and $\mathrm{C}(\mathrm{CtOS})$ show smaller delivery ratios than $S(C t O C)$ and $S$ (Cto $S$ ) because messages are generated by customers and forwarded to other customers who might be about to leave the shopping mall and not have time to forward them. On the other hand, sellers never leave the mall during their working time. For the same sequence of cases the Figure 4 shows the average delay for delivering messages. It seems that the average delay keep increasing following the combinations on the $\mathrm{x}$-axis and as long as messages are generated by customers. These results are roughly the same for each buffer capacity. The average delay improves with bigger buffer capacity when senders and receivers are sellers. When messages are generated by sellers and received by customers, there is trade off between the type and number of intermediate carriers, customers or sellers, and their buffer capacity.

\section{CONCLUSions}

In this paper we provide a method based on the correlation between number of contacts, contact durations and intercontact times of people in shopping mall environments to identify two groups of people, visitors/customers and shopping mall related people, performing different mobility patterns. Our results suggest that communication services in shopping malls might require specific networking protocols. We propose to exploit the two main entities' mobility patterns to route data: customers and shop employees. We evaluate the performance of two semi-Epidemic protocols with our real-world mobility traces as initial study to estimate the effect of delivering messages in shopping mall environments by exclusively forwarding them to customers or sellers, each of which has distinctive mobility patterns. Shopkeepers could form a mobile ad-hoc network backbone and the starting point from which to build wider networks in shopping mall environments. The

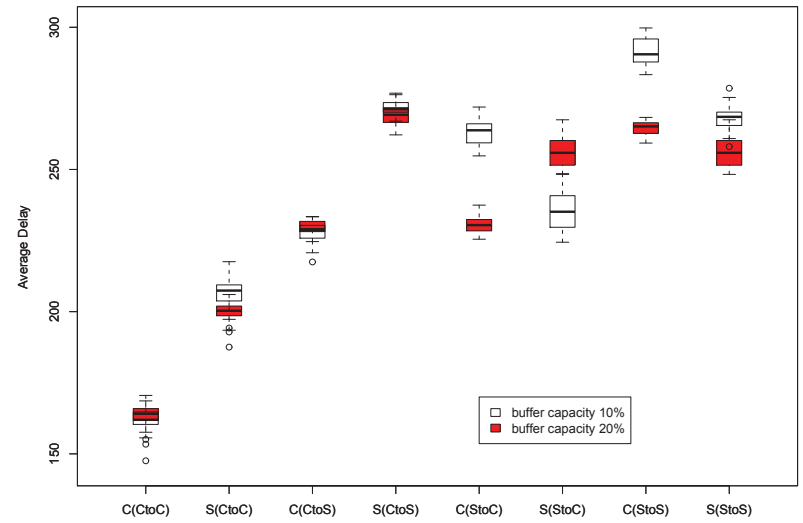

Fig. 4: Boxplot of the average delay for the semi-Epidemic routing protocols.

identification of such groups of people could help greatly in forwarding data. In future work we intend to design and evaluate ad-hoc forwarding algorithms for different pervasive applications in this type of setting, and create realistic mobility models which will accurately represent the observed human mobility patterns.

\section{REFERENCES}

[1] A. Galati and C. Greenhalgh, Human mobility in shopping mall environments, In Proceedings of the Second International Workshop on Mobile Opportunistic Networking, ACM MobiOpp '10, Pisa, Italy, pp. 1-7.

[2] A. Galati and C. Greenhalgh, Exploring Shopping Mall Environment for Ubiquitous Computing, In Proceedings of Ubicomp at a Croassroads, Imperial College London, January 2009.

[3] P. Hui, A. Chaintreau, J. Scott, R. Gass, J. Crowcroft and C. Diot, Pockets Switched Networks and Human Mobility in Conference Environments, In Proceedings of the ACM SIGCOMM workshop on Delay-tolerant networking, pp. 244-251, August 2005 .

[4] A. Chaintreau, P. Hui, J. Crowcroft, C. Diot, R. Gass and J. Scott, Impact of human mobility on the design of opportunistic forwarding algorithms, IEEE Transactions on Mobile Computing, pp. 606-620, June 2007.

[5] N. Eagle and A. Pentland, Reality mining: Sensing complex social systems, Personal and Ubiquitous Computing, Vol. 10, pp. 255-268, 2006.

[6] M. McNett and G. M. Voelker, Access and Mobility of Sireless PDA Users, Technical report, Computer Science and Engineering, 2004.

[7] T. Henderson, D. Kotz and I. Abyzov, The changing usage of a mature campus-wide wireless network, in Proc. of the 10th Int. Conference on Mobile Computing and Networking (MobiCom), pp. 187-201, 2004.

[8] J. Ghosh, M. J. Beal, H. Q. Ngo and C. Qiao, On profiling mobility and predicting locations of campus-wide wireless users, in Proc. of REALMAN '06 Workshop at ACM MobiHoc, pp. 187-201.

[9] F. Benbadis, J. Leguay, V. Borrel, M. Amorim and T. Friedman, Millipede: A Rollerblade Positioning System, ACM MobiCom 2006 - Workshop on Wireless Network Testbeds, Experimental evaluation and CHaracterization (WiNTECH 2006), Los Angeles, USA, September 2006.

[10] P. Hui and J. Crowcroft, Human Mobility Models and Opportunistic Communication System Design, Philosophical Transactions of the Royal Society A: Mathematical, Physical and Engineering Sciences, Volume 366, Number 1872, pp. 2005-2016, June 13, 2008.

[11] V. Vukadinovic and S. Mangold, Opportunistic Wireless Communication in Theme Parks: A Study of Visitors Mobility. In Proceedings of the 6th ACM workshop on Challenged networks, CHANTS '11, pp. 3-8.

[12] V. Lenders, J. Wagner, and M. May, Analyzing the impact of mobility in ad hoc networks, In Proceedings of the ACM SIGMOBILE International Workshop on Multihop Ad Hoc Networks: From Theory to Reality (REALMAN), May 2006, Florence, Italy.

[13] S. Okasha, Altruism, group selection and correlated interaction, The British Journal for the Philosophy of Science, Vol. 56, No. 4, pp. 703 725, December 2005. 